\title{
REFLEXIONES SOBRE LAS POSIBILIDADES DE REFORMAS EDUCATIVAS ORIENTADAS A LA FORMACION UNIVERSITARIA
}

\author{
CONSIDERATIONS ABOUT THE POSSIBILITIES OF COLLEGE EDUCATION REFORMS IN \\ ORDER TO CONTRIBUTE TO THE COLLEGE (UNIVERSITY) FORMATION
}

\author{
Georgina Paulín Pérez*, Julio César Horta Gómez** y Gabriel Antonio Siade Paulínn***
}

\begin{abstract}
RESUMEN
Si se parte del supuesto de que toda Universidad debe y tiene que formar profesionistas útiles, con espíritu de servicio, imaginativos, originales, creativos y productivos, se hace imperativo un diseño curricular que coordine, articule y vincule el pensamiento humanista con el tecnológico (cultura-civilización); la teoría con la empíria (abstracción-concreción); la ética con la práctica (conocimiento acumulado-conocimiento aplicado). Con base en esto, el presente artículo busca: 1) establecer fundamentos reflexivos acerca de la importancia del lenguaje en la educación integral universitaria; y, de manera complementaria 2) hacer visible este problema y cómo combatirlo a partir de la realización de dos módulos (Propedeútico y Concentración Terminal) que, en tanto etapas en la formación universitaria, tratan de generar y desarrollar las competencias necesarias para el mejor desempeño del estudiante en ciencias de la comunicación
\end{abstract}

Palabras clave: formación, competencia-ejecución, semántica, semiótica, hermenéutica.

\begin{abstract}
If it is assumed that all University should and must to educate professionals, service-minded, imaginative, original, creative and productive, it is obligatory to design a curriculum to coordinate, articulate and link humanistic thought with technological (culture-civilization); theory and empiric (abstraction-concreteness); ethics with practice (accumulated knowledge-applied knowledge). Based on this considerations this paper seeks: 1) establish reflexive arguments about the importance of language in the comprehensive university education; and, complementary manner 2) manifest this problem and how to solve it from the realization of two modules (Propedeutic and Concentration Terminal) that, in both stages in university formation commonly seek of generating and develop the competences necessary for the best performance of student in communication sciences
\end{abstract}

Keywords: education (formación), competence-performance, semantics, semiology, hermeneutics.

Fecha de recepción: Mayo 30 de 2014 / Fecha de aceptación: Septiembre 29 de 2014

Tipología: Artículo de Reflexión

Para citar este artículo: Paulín, P.G. \& Horta G.J., \& Siade, P.G. (2014). Reflexiones sobre las Posibilidades de Reformas Educativas orientadas a la Formación Universitaria. Praxis, Vol.10, 134 - 145

*Magister Instituto de Investigaciones Sociales de México, México. E-mail: papg@unam.mx.

**Magister Facultad de Ciencias Políticas y Sociales Universidad Nacional Autónoma de México, México. E-mail: julio_horta@hotmail.com.

*** Magister Instituto de Investigaciones Filosóficas de México, México. E-mail: gsiade@prodigy.net.mx. 


\section{INTRODUCCIÓN}

$\mathrm{E}$ la educación superior universitaria y particularmente en la carrera de Ciencias de la Comunicación de la Universidad Nacional Autónoma de México, las asignaturas de carácter teórico correspondientes al estudio de lenguaje, muestran diferencias y contrastes de modo y contenido en su enseñanza y aprendizaje. En este marco se presenta un doble problema: por un lado, ambigüedades conceptuales en propuestas educativas de planes de estudio en la carrera citada; y por otro, la resistencia de los alumnos al aprendizaje de este tipo de materias.

En efecto, al observar la evolución de los planes y programas de estudio en la carrera de Ciencias de la Comunicación (de la Facultad de Ciencias Políticas y Sociales de la Universidad Nacional Autónoma de México) se puede identificar en las propuestas de las reformas educativas en los últimos treinta años un argumento constante: la innovación de elementos para mejorar la educación. De la misma manera, los diversos diagnósticos realizados en fechas recientes (Centro de Estudios en Ciencias de la Comunicación, 2014), con el objetivo de fundamentar la Reforma a los Planes y Programas de estudio para la Carrera Ciencias de la Comunicación, se evidencian dos problemas fundamentales: por un lado, el carácter problemático en la formación de metodología de la investigación, y por otro, la falta de cualidades de lecto-escritura que permitan una mejor aprehensión de los contenidos dispuestos en la formación básica del estudiante de licenciatura en comunicación.

Sin embargo, el cambio y la novedad no siempre contribuyen con transformaciones progresivas, pues diversos son los factores a considerar para que las propuestas educativas no queden sólo en un plano discursivo, y sobre todo se conviertan en factores regresivos de la educación. En el caso particular de los planes de estudio citados se presenta, por ejemplo, tanto una ambigüedad conceptual en los enfoques como en los sentidos pedagógicos de las propuestas: y en el ejercicio docente se nota inercia y resistencia en los profesores a prácticas que difieran de las formas establecidas de trabajo; y finalmente en el desempeño de los estudiantes problemas de competencia cognitiva y de ejecución. En este contexto, el cuerpo del presente artículo de reflexión está constituido por un esbozo preliminar de los datos recolectados en las propuestas educativas de planes de estudio en la carrera citada; así como por los supuestos derivados de investigaciones previas realizadas por los autores del presente artículo. Así, para poder establecer los fundamentos acerca de la importancia del lenguaje en la educación universitaria, se proponen asignaturas que de manera articulada, permitan la formación integral del estudiante universitario.

Luego, este trabajo buscará dar cuenta de los puntos relacionados con la "ambigüedad conceptual" y con el "desempeño de los estudiantes". Específicamente el problema sobre el "ejercicio docente" se abordará en un trabajo posterior. Esta decisión responde a una cuestión metodológica: a saber, consideramos que dentro de una propuesta de reforma educativa es pertinente, en principio, dar cuenta de los tópicos implicados en la definición de educación y en el sujeto que se busca educar; para, posteriormente, trabajar una propuesta de modelo pedagógico que sugiera directrices en las prácticas docentes. Pues, consideramos que la relación educación-educando es un punto inicial en la reflexión sobre una reforma educativa. Si bien esto no responde a un criterio jerárquico, sí es importante hacer notar que esta relación es una condición necesaria e inicial: pues una reforma educativa tiene como fundamento una noción acerca de qué es la educación; y en este sentido, debe poder dar respuesta a los objetivos de aquello que se busca formar (sujeto-educando). Por tanto, determinar el carácter y viabilidad de las prácticas docentes responde a un momento que, aunque necesario también, debe cimentarse sobre la base de la relación educación-educando.

\section{METODOLOGÍA}

Si bien en este trabajo se busca reflexionar sobre la pertinencia de una propuesta de plan de estudios a nivel universitario, empero el problema que sustenta esta reflexión es la inconsistencia 
discursiva de los contenidos semánticos en relación con dos categorías fundamentales: a saber, formación-competencia. En la función docente y las repercusiones de dicha inconsistencia en el desempeño formativo de los estudiantes. De ahí que, debe entenderse este trabajo como un ejercicio especulativo, que busca dar cuenta de un problema léxico-semántico implicado en la reforma educativa. Pero también tiene una dimensión propositiva, la cual está orientada (como hemos señalado ya en otros trabajos) a la "educación entitativo funcional... (Para) la actualización del razonamiento humano y consecuentemente la operatividad de la facultad del intelecto y de la voluntad... (Mediante) la enseñanza del lenguaje... pues su aprendizaje uso y aplicación posibilita la observación analítica de las cosas, mediante la aprehensión, la nominación, la relación, la crítica, las cuales a su vez estimulan la facultad de la imaginación” (Paulín, Horta, Sánchez, Siade \& Jiménez, 2012).

Antes de abordar las cuestiones implicadas en el problema léxico-semántico y su consecuente ambigüedad conceptual, resulta pertinente señalar que el presente artículo está circunscrito a la metodología de un trabajo de investigación más amplio. Como punto inicial para realizar esta exploración, entre los antecedentes que fundaron las premisas teóricas se puede señalar en primera instancia la investigación teóricacualitativa acerca de los objetos significantes y sus relaciones estructurales léxico conceptuales (en el discurso tradicional de la UNAM), y una línea de corte empírico-cuantitativo sobre las valoraciones de entrevistados (sujetos-académicos) hacia los objetos significantes del mundo universitario que se publicó como libro bajo el título Humanidades y Universidad. La UNAM desde una intertextualidad humanística (Paulín, Horta, \& Siade, 2012).

En dicho trabajo, específicamente en el Capítulo II, se desarrolla con mayor amplitud los métodos de análisis involucrados en la exploración cuantitativa y cualitativa que sustenta el análisis, reflexión y propuesta del presente artículo. Ahora bien, en el estudio cualitativo, los procedimientos que se siguieron e instrumentos que se emplearon en la recolección de datos fueron:
1. Método supra-lingüístico o análisis mediante categorías temáticas (categorías filosóficas de causalidad), para ordenar los segmentos del texto en las fuentes consultadas

2. Adaptación del modelo Quillian (Modelo Q) para la representación de las connotaciones convencionalmente atribuidas a lexemas específicos (Universidad, Formación, Competencia, etcétera).

3. Métodos o análisis supra lingüísticos para la identificación de palabras, frases, y temas, se seleccionan enunciados de los documentos historiográficos consultados que manifiestan significantes con contenidos del discurso humanista de la Universidad Nacional Autónoma de México (Legislación Universitaria, Estatutos, Planes de Estudio, Historias Oficiales, etcétera). Y análisis infra-lingüístico, para seleccionar formas significantes cuyos contenidos culturales se repiten (núcleos) diacrónicamente en la descripción historiográfica de las fuentes consultadas.

Por otro lado, en la exploración cuantitativa el instrumento que se utilizó fue un cuestionario de opción múltiple (aplicado a profesores, investigadores y estudiantes) para la obtención de datos, cuyos resultados se validaron con las medidas de estadística descriptiva, inferencial en un análisis factorial de varianzas (ANOVA) y de correlación y similaridad Pearson.

Además, es importante señalar que este trabajo se inscribe en un proyecto rector, titulado Propuesta de Neo-humanidades: problemática de conjunciones, que coordina la investigadora G.Paulín P. y en el que colaboran J.Horta G. Y G.Siade P., en el Instituto de Investigaciones Sociales (UNAM). Además -derivado del anterior - en el proyecto la Dimensión pragmática de las Neo-humanidades se han realizado indagaciones en el espacio de la función educativa universitaria y cuyos frutos han sido diversas publicaciones, entre las que citamos Reflexiones sobre la importancia del lenguaje en la educación entitativo-funcional universitaria; Retos de la educación superior universitaria: la enseñanza del lenguaje en la carrera de Ciencias de la Comunicación; Formación-competencia: zun 
binomio articulador de tradición-modernidad? En el contexto de la educación universitaria, entre otros. Todo este trabajo de indagación, exploración y reflexión nutren, fundamentan, y orientan las reflexiones y propuestas de este artículo.

En este ámbito de investigación, se advierte reiteradamente un problema léxico-semántico, que ha abierto otra vía de exploración materializada en el texto Homologías Nocionales en la resignificación Formación-Competencia: repercusiones en la educación universitaria, el cual está en proceso de desarrollo. En el trabajo citado identificamos al menos tres directrices del problema léxicosemántico: por un lado, la relación de homología entre términos, en donde sentidos diferentes están vinculados por su relación con un mismo objeto de referencia o un proceso común (a saber, la educación humana, o de lo humano); por otro, está la relación a-tópica, en donde el cambio de espacio semántico de los términos permite problemáticamente la generalización, imposición y uso de los conceptos en ámbitos educativos que resultan ajenos al contexto de origen.

Una tercera vía de este problema está en la construcción de términos vacíos, pues si bien la palabras y conceptos tienen sentido en el contexto de uso, empero carecen de significado, debido a que la relación con su referente es problemática -incluso en algunos casos puede ser contradictoria. En este sentido, los vínculos significativos entre el referente y el término significante sólo se establecen accidentalmente con algún atributo o cualidad predicado en la definición referente, dejando las demás cualidades fuera del espacio semántico de sentido. Un caso de este tipo podemos rastrearlo en las denominaciones acerca de la palabra Universidad. Las diferentes instituciones que se autonombran Universidades no se corresponden, en sus funciones, con los atributos predicables de la definición del término. Salvo el atributo específico de "profesionalización”. Así pues, las instituciones que otorgan un grado de licenciatura para desempeñarse en el ámbito laboral se denominan contemporáneamente como Universidades, aunque la formación que ahí se imparte no cumpla con las funciones y atributos implicados en el concepto.
En particular, nos hemos concentrado, precisamente, en las homologías nocionales que se estructuran en la resignificación de los conceptos. Esta especificidad semántica es interesante pues nos permite ver cómo hay ciertas continuidades de la raíz léxica de los términos, que los vinculan con un mismo referente; pero que, en una dirección contraria, derivan en sentidos diferentes. Por ello, al deformar sus contenidos dan lugar a problemas epistemológicos cuya repercusión en las prácticas docentes origina problemas de carácter ontológico.

En relación con la a-topía (que en su significado etimológico se refiere a "sin lugar") se alude a un proceso semántico específico: a saber, el cambio de espacio significativo en los términos, conceptos y palabras; donde el sentido de las expresiones verbales está determinado por un contexto semántico diferente al contexto de origen. Como consecuencia del proceso de globalización, el sentido de los signos se generaliza hacia diferentes contextos, estabilizando diversos significados que son en principio ajenos al espacio semántico de origen.

En general, todo este espacio de indagación nos ha permitido reflexionar sobre la posibilidad de una reforma educativa en la formación universitaria, a pesar de la coacción de intereses que buscan encausar las prácticas educativas hacia la capacitación. Es en esta dinámica donde surgen, como efecto de una imposición léxica, las ambigüedades conceptuales que resultan problemáticas al momento de perfilar los fines y objetivos de la educación universitaria.

\section{Ambigüedad conceptual en los planes de estudio y en los sentidos pedagógicos de las propuestas.}

En relación con el problema conceptual, las diversas propuestas planteadas en planes y programas de estudio en Ciencias de la Comunicación evidencian orientaciones que tienden a subrayar la adquisición de conocimiento; otras que enfatizan lo utilitario, en el sentido de resolver problemas, y finalmente aquellas que 
puntualizan capacidades y destrezas laborales. El manejo de esta triada conceptual se vuelve problemática al considerar el contexto semántico donde se codifica el significado y sentido del concepto Universidad, y a la luz del cual se entienden las diferentes formas de realizar actividades universitarias.

En efecto, la informacion documentada fundamentalmente en Corominas y Pascual (1991), la Real Academia Española (1984) y Martín (1968); permitió precisar aspectos constitutivos y rasgos esenciales del objeto significante Universidad, a la luz de los cuales Paulín et al. (2012) establecen la relación intelectual de conceptos que les permite afirmar proposiciones tales como: los rasgos que fluyen de la esencia de (su) naturaleza universal son por una parte, la Universalidad, pues el carácter de este término es su sentido de universal como objeto de la inteligencia, admite dos sentidos, entendido como universo de conocimiento, y en tanto saber de totalidad, de ahí el origen del Espíritu del Saber. Por otra parte, el sentido de Comunidad, como congregación que forma cuerpo de maestros-discípulos (Espíritu de Cuerpo), en tanto transmisión y transformación del saber mediante la formación-cultura (cultivo) de sus congregados, de donde la fuente del Espíritu Escolástico.

Estas consideraciones sobre el valor etimologico de la palabra Universidad se sustentan, complementan y fortalecen con definiciones reales como la de Kant (1963) y Heidegger (1989) que resaltan el concepto Ciencia, o el de Ortega \&Gasset (1968) que subraya el de Educacion, y tambien las argumentaciones de Bueno (1960) y Uribe (1984) sobre el Humanismo Cultural como basamento de su reflexion sobre el objeto Universidad (por citar algunos de los autores consultados). De ese contexto conceptual Paulín et al. (2012) derivaron afirmaciones de la Universidad como una Institución Cultural del Saber y una Institución forjadora de Cultura Superior que por supuesto contraria la aseveración que contempla la Universidad como una institución que capacita, pues en tanto que solo capacite, no puede ser Universidad; aunque la Universidad que capacita puede ser Universidad por lo que en ella no es capacitar.
Por eso, cuando se vinculan conceptos contrapuestos en un mismo enunciado su interpretación ambigua repercute en la normatividad que reglamenta funciones de la institución y que orienta la actividad de sus miembros. Tal sucede en el texto del artículo 1 de la Ley Orgánica de la UNAM de 1945, donde se expresan los fines de la Universidad Nacional Autónoma de México, y que a la letra dice: “(...) que tiene por fines impartir educación superior para formar profesionistas, investigadores, profesores universitarios y técnicos, útiles a la sociedad" (Universidad Nacional Autonoma de Mexico, 1991).

Pero, más allá de la lectura lineal de los enunciados explícitos en la Legislación Universitaria, amerita detenerse en los vocablos formaciónutilidad. La voz formación se ha interpretado tradicionalmente en el contexto del conocimiento racional que se identifica con el conocimiento universalmente válido; en tanto, el vocablo utilidad se inscribe dentro del ámbito práctico, del conocimiento intuitivo que representa algo subjetivo, y aunque es posible su objetividad, ésta no implica validez universal. De suerte que si en la Legislación se usa el significante formación tendría que ser consecuente con la cualidad que pretende conferirle al educando, que en cuyo caso sería la racionalidad (la cultura). Pero "si la cualidad es la utilidad, entonces no estaría formando, sino capacitando, y en tal sentido, la educación orientada a la realización del potencial humano y a su búsqueda por la verdad, se convierte en instrumento al servicio de las necesidades de un sistema social en una época y momento específicos para fines determinados espacial y temporalmente." (Paulín et al., 2012, p. 231).

Esta ambigüedad conceptual se advierte en las propuestas de modificación de los planes de estudio (1996-2000-2007) de Ciencias de la Comunicación, en cuyo texto se hace evidente un discurso de cambio que busca superar los planes anteriores (1976) para satisfacer las necesidades del mercado laboral. No obstante, a pesar de que en la exposición de motivos se descalifica lo anterior para validar las bondades de lo nuevo, se mantienen las referencias conceptuales formación-capacitación, como 
puede advertirse en las siguientes citas del plan de estudios de la licenciatura en Ciencias de la Comunicación: "La transformación social, económica y política, tanto a nivel nacional como internacional...desde principios de los años ochenta...contribuyó a la desactualización de un plan de estudios...que estaba centrado más en la formación de investigadores y analistas sociales...Todo esto coadyuvó a la marginación de una formación profesionalizante que el mercado laboral ha venido exigiendo[...] dada la nueva división internacional de trabajo, es necesario contemplar la generación de nuevas competencias y habilidades acordes con las exigencias internacionales[...]. En relación con el perfil del egresado se puntualiza que deberá contar con una sólida formación social y humanística...asimismo con una formación teórico, metodológica y técnica en el campo de la comunicación que le permitirá comprender, explicar, analizar, criticar y teorizar"... (Centro de Estudios en Ciencias de la Comunicacion, 1998, pp. 4-20).

Sin embargo, en los programas no se precisan las competencias profesionales que son objeto de formación y de capacitación, ni los mecanismos y etapas en los cuales se realizaría esa formación y esa capacitación para comprender, explicar, analizar, criticar y teorizar. En realidad, lo que se advierte es un Plan de estudios donde predomina el criterio de la carrera de comunicación como profesión sobre su concepción como ámbito disciplinario, lo que repercute en la duplicación de temas-fenómenos que lo mismo se tratan en talleres o en las asignaturas que debieran nutrirlos; esta confusión, ambigüedad y desarticulación se advierte también en las designaciones de las asignaturas (unas veces se opta por el temafenómeno y otras por la disciplina), de la misma manera los contenidos de los programas se presentan de manera atomizada (un mismo tema puede ser tratado en taller, metodología o teoría; como objeto de taller en teoría o como objeto de teoría en taller, como investigación o como técnica de investigación), entre otros problemas evidentes por la falta de criterios articuladores de los Planes y Programas de Estudio.
Problemas de competencia cognitiva y de ejecución en el desempeño de los estudiantes.

Por otra parte, y de acuerdo a los resultados de la observación en el espacio del aula se identifica - entre los estudiantes- “...dificultades de análisis y abstracción; retención efímera de lo inmediato y concreto; práctica del comentario; priorización de la obtención de calificación por encima del conocimiento; problemas en el manejo sintáctico y gramatical del lenguaje: repetición de términos tradicionales que imposibilitan la aprehensión de nuevos sentidos y conceptos que ofrecen las teorías contemporáneas; desinterés por la búsqueda de conocimiento articulado y lectura de textos completos, así como, incapacidad de articulación de los contenidos temáticos; pasividad en el aprendizaje, ya que los tiempos para abordar los temas del curso se limitan a las horas de clase y los contenidos a las síntesis ofrecidas por el docente." (Paulín, Horta, Siade \& Jiménez, 2013, p. 6)

Por supuesto la resistencia de los estudiantes para desarrollar hábitos operativos del intelecto es uno de los problemas originado por la ineficacia del Sistema Nacional de Educación que se nutre de los parámetros educativos de la "racionalidad mercantil" contemporánea. Sin embargo no puede soslayarse el hecho de que Planes y Programas de Estudio Universitarios, en la Facultad de Ciencias Políticas y Sociales y particularmente en la carrera de Ciencias de la Comunicación, representan un contexto problemático para la formación universitaria. En efecto, las concepciones, los intereses y los propósitos que sustentan el perfil de los profesionistas y la estructuración de la carrera no siempre coinciden con el ámbito propio de cada disciplina o con el de los fenómenos que se abordan en las asignaturas. De la misma manera, no es clara la colindancia entre el propósito de formar profesionistas universitarios (no técnicos), con los fines generales vertidos en la Legislación Universitaria y con la metodología de la enseñanza orientada a la articulación de los ámbitos de aprendizaje: cognitivo-normativo-técnico.

\section{Reflexiones sobre las posibilidades de una}




\section{propuesta de reforma consecuente con la formación integral universitaria}

Si se parte del supuesto de que la Universidad debe y tiene que formar profesionistas útiles, con espíritu de servicio, imaginativos, originales, creativos y productivos, se hace imperativo un diseño curricular que coordine, articule y vincule el pensamiento humanista con el tecnológico (cultura-civilización); la teoría con la empiria (abstracción-concreción); la ética con la práctica (conocimiento acumulado-conocimiento aplicado).

Con base en esto, la reflexión (sobre las posibilidades de una propuesta de reforma consecuente) busca de manera complementaria dar cuenta de este problema formativo a partir de la realización de dos módulos (Propedeútico y Concentración Terminal) que, en tanto etapas en la formación universitaria, tratan de generar y desarrollar las competencias necesarias para el mejor desempeño del estudiante en ciencias de la comunicación.

El planteamiento de estas dos etapas parte de un supuesto importante, a saber, que el estudiante en ciencias de la comunicación tiene como objeto de estudio el lenguaje en sus diferentes dimensiones, desde lo sintáctico hasta lo social. Si bien puede argüirse que el objeto de estudio es la comunicación misma, hay que reconocer que ésta, en sus diferentes momentos de realización, implica el uso y comprensión del alguna forma de lenguaje, o bien de alguna manifestación sígnica (sea objetiva como la materialidad misma del signo, o bien puramente subjetiva, como los sujetos inmersos en su dinámica social y cultural).

Por ello, no puede dejar de reconocerse que es el lenguaje el objeto de estudio que implica toda investigación en comunicación. Pero, además, es la herramienta con la cual el comunicador trabaja en su desempeño profesional: pues en sus diferentes ámbitos de realización laboral y profesional (periodismo, publicidad, organizacional, producción visual, producción audiovisual, etcétera), el comunicador se ve en la necesidad de entender la dinámica de un lenguaje particular; y, sobre todo, se ve en la obligación de conocer y dominar la lógica interna de ese lenguaje para poder construir y crear expresiones concretas. En este sentido, la presente propuesta se justifica sobre la base de necesidades profesionales y de investigación que se evidencian en el quehacer cotidiano del comunicólogo y del comunicador. La diversidad lingual, potenciada hoy día por los medios electrónicos y antaño circunscrita al ámbito normativo de las diversas ramas gramaticales (Lingüística, Sociolingüística, Gramática...), representa ahora un terreno de exploración crucial para el estudioso de las Ciencias de la Comunicación, que busca adquirir la capacidad de producir y comprender, competente y eficazmente, mensajes bien formados, interpretables, y apropiados a la situación comunicativa donde desempeñe su actividad profesional. Al comunicólogo se le demanda competencia en la comunicación, lo cual quiere decir que en términos teóricos debe poder identificar causas de las modalidades comunicativas y anticipar efectos sociales deseables e indeseables de las mismas. Pero, también tiene que ser hábil ejecutante, capaz de obtener eficacia en su intervención como orador, como redactor, como escritor, como locutor-comentarista, en términos profesionales.

De ahí la importancia de contar con los instrumentos indispensables necesarios para el análisis y la construcción del discurso oral y escrito, y tener así la posibilidad de aprender y valorar la propia oralidad (posibles locutores profesionales), de contrastarla y complementarla con la propia literalidad (redactores periodísticos o de otro tipo), de explorar y aprovechar las posibilidades de uno de los "medios" frente a otro, utilizando las estrategias pertinentes para el medio específico, la situación comunicativa concreta, la intención y propósitos buscados.

En este sentido, se exploran propuestas sobre materias: unas, con carácter obligatorio para desarrollar las competencias necesarias (proyectivas-propositivas; socializantes; moralizantes; comunicativas) que a priori necesita el estudiante de Ciencias de la Comunicación (espacio de nuestra actividad docente) antes de ingresar a la formación básica de la licenciatura; y, otras (como "opción terminal") que posibiliten la ejecución de esas competencias para analizar 
y comprender los diferentes fenómenos comunicativos, con base en la indagación acerca del funcionamiento de los componentes sígnicos que constituyen los diferentes sistemas de lenguaje.

Dentro de este marco referencial, la propuesta de reforma a los planes y programas de estudio tiene como finalidad formar a los estudiantes en ciencias de la comunicación en cuatro ámbitos de realización, que son complementarios y necesarios para el desempeño profesional del comunicólogo y del comunicador, dentro del proceso de aprendizaje cuya finalidad es enseñar al estudiante a "saber-hacer-bien". El primero de estos ámbitos a considerar es el lógico-discursivo, en donde el estudiante adquiere las herramientas necesarias para el entendimiento de los argumentos, contra-argumentos, y proposiciones derivadas que le permiten acercarse no sólo a la comprensión de textos, sino además le permite tener una estructura mental que posibilita el mejoramiento en la expresión (oral o escrita) de ideas y el desarrollo de planteamientos y problemas de investigación.

De manera complementaria, se considera el ámbito ético, en donde se busca sensibilizar al alumno hacia los problemas sociales que le son propios y de interés; así como a reconocer los procesos históricos implicados en dichos problemas. De ahí que, además, se busca desarrollar en el estudiante de ciencias de la comunicación la sensibilidad estética, capaz de propiciar en los estudiantes la capacidad creativa para proponer soluciones concretas a cuestiones sociales específicas.

Finalmente, el último ámbito implicado y que resulta necesario considerar en el desarrollo del estudiante en ciencias de la comunicación (en particular) es el ámbito técnico: en donde se les proporcionará el manejo de instrumentos y tecnologías indispensables tanto para los procesos de investigación social, como para el desempeño profesional en los diferentes espacios laborales.

Como consecuencia, el proceso efectivo de estos ámbitos permitirá el desarrollo de diferentes competencias que serán de suma importancia en la formación y desempeño tanto de comunicadores como de comunicólogos. Algunas de las cuales se consideran a continuación:

Competencias Naturales: Proyectivaspropositivas; Competencias derivadas: selectiva, reflexiva, auto-determinativa; Instrumentos: Hábitos operativos directivos, imperativos, ejecutivos.

Competencia Natural: socializante; Competencia derivada: interactiva; Instrumentos: Hábitos operativos para el aprendizaje referido a los demás: tolerancia, comprensión, ayuda, urbanidad.

Competencia natural: moralizante; Competencias derivadas: autodominio, responsabilidad; Instrumentos: Hábitos operativos para el aprendizaje referido a valores en las opciones de perfeccionamiento, en el de las preferencias y en el de la convivencia.

Competencia natural: comunicativa; Competencias derivadas: transmisión, interpretación, entendimiento, comprensión (de saberes); Instrumentos: Hábitos operativos para el aprendizaje de códigos y referentes comunes.

\section{RESULTADOS Y DISCUSIÓN}

Los resultados sobre una propuesta de reforma a los planes y programas de estudio lleva a la discusion de los planteamientos y justificacion de las dos etapas que se contemplan en el presente trabajo: la correspondiente a la propedeútica y asimismo a la etapa terminal.

\section{Primera etapa: propedéutico}

La formación básica en ciencias sociales, y en particular en ciencias de la comunicación, exige competencias y habilidades que posibiliten al estudiante analizar y comprender problemas teóricos planteados desde diferentes posturas de pensamiento; así como problemas sociales vistos desde un punto teórico de anclaje. La comprensión y análisis implican, además, las competencias necesarias para poder desarrollar, sostener, y argumentar una postura teórica; pero sobre todo obliga a tener las habilidades que 
permitan explicar hechos y problemas sociales desde el análisis y la teoría.

Uno de los problemas mostrados en los diagnósticos de la licenciatura en Ciencias de la Comunicación (Centro de Estudios en Ciencias de la Comunicación, 2014) es la falta de habilidades de lecto-escritura. La explicación y justificación de este problema se concentra en las carencias de la educación básica y las ausencias educativas en la educación medio superior. Sin embargo, del reconocimiento de este problema formativo no se sigue su consecuente aceptación. En aras de una formación integral y óptima, en la cual se realicen los objetivos académicos propuestos para la formación de los estudiantes de licenciatura en ciencias de la comunicación, se propone realizar un curso propedéutico de un semestre.

La idea de un curso propedéutico responde a dos objetivos fundamentales: por un lado, preparar formativamente a los candidatos a estudiantes de licenciatura en ciencias de la comunicación, para que adquieran las habilidades de lecto-escritura necesarias para el buen desempeño universitario; por otro, evidenciar a los estudiantes que pueden y quieren desarrollar los contenidos teóricos y metodológicos implicados en los contenidos formativos de la licenciatura.

En principio, el curso se propone como una instancia obligatoria, y en consecuencia, sólo podrán ingresar a la licenciatura los aspirantes que hayan aprobado el curso. La razón es relativamente sencilla y consecuente: sólo aquellos que hayan mostrado tener las competencias mínimas y necesarias, pueden desarrollar de mejor manera la licenciatura. Este planteamiento no es en modo alguno excluyente sino, más bien, selectivo: busca establecer las bases para poder elegir a los estudiantes que tengan las aptitudes y disposiciones que les permitan desarrollar la formación académica en ciencias de la comunicación.

En este sentido, se proponen materias con carácter obligatorio para desarrollar las competencias necesarias que a priori necesita el estudiante de ciencias de la comunicación antes de ingresar a la formación básica de la licenciatura. Las materias responden a las necesidades formativas implicadas en tres grupos: habilidades de lectura (análisis), habilidades de escritura (síntesis), y habilidades de lecto-escritura (analítico-sintéticas).

\section{Segunda etapa: área de concentración terminal: estudios del lenguaje (semiótica y hermenéutica)}

En principio es pertinente señalar que los autores y textos que orientaron estas reflexiones fueron; en semiótica: "Eco, Umberto" (2000), (1992), (1995); "Peirce, Ch. S." (1997); "Magariños M." (1996); en hermeneútica: "Beuchot, M." (2011), (2005); "Ricoeur, P.” (2001); Gadamer, H. G.” (2012).

En cuanto a la presente propuesta, ésta se circunscribe al planteamiento general esbozado anteriormente: a saber, que el estudiante en ciencias de la comunicación tiene como objeto de estudio el lenguaje en sus diferentes dimensiones, desde lo sintáctico hasta lo social. Si bien puede argüirse que el objeto de estudio es la comunicación misma, hay que reconocer que ésta, en sus diferentes momentos de realización, implica el uso y comprensión del alguna forma de lenguaje, o bien de alguna manifestación sígnica (sea objetiva como la materialidad misma del signo, o bien puramente subjetiva, como los sujetos inmersos en su dinámica social y cultural).

Por tanto, la enseñanza del lenguaje, desde la perspectiva de la formación profesional del estudiante de comunicación, plantea la necesidad de organizar y articular los conocimientos de las diversas disciplinas que se ocupan de su estudio teórico, y vincular este saber con los conocimientos y habilidades de las prácticas comunicativas que singularizan su formación profesional.

En las últimas décadas en la carrera de Comunicación, de la Universidad Nacional Autónoma de México, se ha experimentado una constante en la tendencia negativa de los 
estudiantes para aprender tópicos relativos al lenguaje, la lengua y a las variantes linguales. Pero, si bien el contrasentido de tal estudio se dio o reforzó cuando sólo se puntualizaba lo lingüístico, su sentido comenzó a recuperarse al considerar, preferentemente, la vertiente comunicativa del lenguaje: pues en este horizonte se hacen evidentes las posibilidades del lenguaje para convertir las impresiones en expresiones y en virtud de la comunicación compartirlas, logrando con ello la comunidad vivencial empática que da sentido a las acciones humanas que hacen de ellas una sociedad.

Por consiguiente, el interés por tales temas (lenguaje y comunicación) surge de las posibilidades que ofrecen para entender el lenguaje y la lengua, como instrumentos y fenómenos que sustentan, facilitan, obstruyen, impiden, in-forman, con-forman o de-forman significados, sentidos, conceptos y valores, al tiempo que orientan las conductas entre humanos.

De ahí que es de vital importancia que el estudiante en ciencias de la comunicación tenga como "opción terminal" la posibilidad de formarse en el estudio del lenguaje, como espacio académico que potencialmente les permita analizar y comprender los diferentes fenómenos comunicativos, con base en la indagación acerca del funcionamiento de los componentes sígnicos que constituyen los diferentes sistemas de lenguaje.

Si bien esta propuesta parece, en principio, contraria a los intereses de los estudiantes (pues en algunos diagnósticos las materias del eje de lenguaje han sido las de mayor índice de reprobación), empero el interés de proponer el estudio del lenguaje como área terminal responde al beneficio formativo de los estudiantes: a saber, contar con una formación rigurosa que les permita desempeñarse, no sólo en el campo de investigación en comunicación, sino además tener un mejor desempeño (analítico y reflexivo) en las actividades profesionales dentro del campo laboral.

El objetivo de la propuesta de una nueva opción terminal enfocada en el estudio del lenguaje consiste en contemplar dentro de esta área posibles estrategias didácticas para estimular hábitos operativos que permitan al estudiante comprender, explicar, analizar, criticar y teorizar, acerca del fenómeno comunicativo.

De manera general, los ejes que articularán la formación y desarrollo del estudiante en esta opción terminal están en razón de las posturas teórico-metodológicas que provienen tanto de la semiótica como de la hermenéutica. Así pues, los ámbitos de conocimiento implicados y las materias dispuestas estarán circunscritos a los intereses formativos de las áreas consideradas en el estudio del lenguaje.

La idea de circunscribir el estudio del lenguaje a estas dos áreas tiene el propósito de vincular dos niveles de observación en los procesos comunicativos: por un lado, el análisis semiótico (a nivel sintáctico, semántico y pragmático) que permitirá entender los procesos comunicativos a partir de la productivo.

En esta dirección, "la enseñanza del lenguaje es primordial para los estudiantes de todas las carreras universitarias; y, particularmente para aquellos interesados en la comprensión e interpretación de los fenómenos humanos contemplados como sistemas de significación que se organizan en estructuras ordenadas de acuerdo con reglas que permiten generar funciones semióticas, mediante las cuales un objeto y un sujeto se convierten en signo de sus usos o funciones posibles. Sin embargo, para lograr este enfoque integrador se requiere competencia y ejecución lingüística, cognitiva y el desarrollo de hábitos operativos del intelecto." (Paulín, Horta, Sánchez, Siade \& Jiménez, 2012, p. 138).

Los temas y propuestas presentados en este trabajo, merecen un análisis más detallado para poder determinar sus posibilidades, empero es una aproximación al enfoque del estudio del lenguaje, no sólo en su función instrumental, sino como un fenómeno socio-cultural que identifica a las comunidades y orienta sus modos de vivir, pensar y sentir el mundo humano, así como un espacio propicio a las formación, transformación, traducción y uso de los diversos sistemas 
de lenguaje; y por otro, la síntesis hermenéutica, en la cual se comprenderán los fenómenos comunicativos en razón del contexto histórico, la inter-subjetividad y los procesos dialécticos implicados.

De ahí que las relaciones entre semiótica y hermenéutica permiten establecer cuatro dimensiones de estudio y, de manera consecuente, tres ámbitos de aprendizaje. A saber:

Dimensiones de estudio...

- Sintáctica: coherencia entre los signos

- Semántica: adecuación entre los signos y aquello que representan

- Pragmática: vínculo entre los signos, aquello que representan y quienes usan los signos para expresar una realidad)

- Interpretativa: vínculo de los hablantes entre sí.

- Ámbitos de aprendizaje...

- Técnico: ejecución en el hablar y escribir; dimensión del "hacer".

- Cognitivo: competencia lógica; dimensión del "saber hacer".

- Normativo-Valorativo: competencia éticovivencial; dimensión del "saber hacer bien".

Sin embargo, es importante reconocer que la diversidad de campos de estudio que se abren a partir de estas dos áreas permite conformar un espacio de desarrollo necesario para el estudiante de Ciencias de la Comunicación. El análisis semiótico y la comprensión hermenéutica disponen de herramientas teórico-metodológicas que permiten la interpretación rigurosa de hechos sociales; pero, además, posibilitan las competencias creativas del estudiante, pues permiten comprender-entender la lógica de un lenguaje y, desde ahí, potenciar el uso consciente y creativo de las posibles transformaciones que permite un determinado sistema de signos en la construcción de textos concretos.

En este sentido, se plantean dos ámbitos particulares que responden a las necesidades formativas teóricas (comunicólogo) y prácticas (comunicador) de la carrera Ciencias de la Comunicación. A partir de estas necesidades se dispone una distinción entre un eje de materias obligatorias, que buscan formar desde la teoría las competencias que generan hábitos analítico-sintéticos; y otro eje de materias optativas, en las cuales se desarrollen estrategias pedagógicas que vinculen la teoría con la práctica profesional en áreas específicas de interés para el comunicador.

\section{CONCLUSIÓN}

Las reformas de la universidad no pueden circunscribirse a problemas financieros y tecnológicos; tampoco a cambios estructurales de la normatividad, pues mantener y revitalizar la formación integral dignificadora de lo humano que deriva precisamente de la esencia de la Universidad, hace imperativo la necesidad de cuestionar la influencia de las corrientes contemporáneas, cuya ideología tecnicista sostiene las ideas de modernización y desarrollo como metas de mejoramiento humano y particularmente la de una educación centrada en la capacitación de destrezas productivas y habilidades técnicas, para legitimar su necesidad de disponer capital humano funcional, útil y transformaciones de todas las dimensiones de la actividad humana.

\section{REFERENCIAS BIBLIOGRÁFICAS}

Beuchot, M. (2005).Tratado de Hermenéutica analógica: hacia un nuevo modelo de interpretación. México: UNAM.

Beuchot, M. (2011). Epistemología y Hermenéutica Analógica. México: Universidad Autónoma de San Luis.

Bueno, M. (1960). Humanismo y universidad. México: IIS/UNAM.

Centro de Estudios en Ciencias de la Comunicación. (1998). Plan de Estudios de la Licenciatura en Ciencias de la Comunicación. México: Facultad de Ciencias Políticas y Sociales-UNAM.

Centro de Estudios en Ciencias de la Comunicación. (2014). Diagnóstico de la Licenciatura en Ciencias de la Comunicación. México: Facultad de Ciencias Políticas y Sociales. UNAM.

Corominas, J., Pascual, J.A. (1991). Diccionario crítico etimológico, castellano e hispánico. Madrid: Gredos.

Eco, U. (1992). Los límites de la interpretación. Barcelona: Lumen. 
Eco, U. (1995). Interpretación y sobreinterpretación. Reino Unido: Cambridge University Press.

Eco, U. (2000). Tratado de Semiótica general. Barcelona: Nueva Imagen.

Gadamer, H.G. (2012). Verdad y Método. Salamanca: Ediciones Sígueme

Heidegger, M. (1989). La autoafirmación de la universidad alemana. El rectorado, 1933-1934. España: Tecnos.

Kant, E. (1963). El conflicto de las facultades. Buenos Aires: Losada.

Magariños, J. A. (1983). El signo: las fuentes teóricas de la semiología. Buenos Aires: Hachette.

Magariños, J. A. (1996). Los fundamentos lógicos de la semiótica y su práctica. Buenos Aires: Edical.

Martín, A. (1968). Enciclopedia del idioma. Madrid: Aguilar.

Ortega \& Gasset, J. (1968). Misión de la universidad. Madrid, Revista de Occidente.

Paulín, G., Horta, J., \& Siade, G. (2012) Humanidades y Universidad: La UNAM desde una intertextualidad humanística. México: Fontamara.
Paulin, G., Horta, J., Sanchez, L., Siade, G., \& Jiménez, G. (2012). Reflexiones sobre la importancia del lenguaje en la educación entitativo-funcional universitaria. Enunciación. 17 (1). pp. 120-139. Enero-Junio 2012. Bogota, Colombia.

Paulín, G., Horta, J., Siade, G., \& Jiménez, G. (2013). Retos de la educación superior universitaria: la enseñanza del lenguaje en la Carrera Ciencias de la Comunicación (Universidad Nacional Autónoma de México). en Revista Iberoamericana de Estudios de Oralidad, Vol. 1 (1), 1-26, recuperado de: http//revistaoralidad.wix.com/iberoamericana en trámites.

Peirce, S. (1997). Escritos Filosóficos. México: Colegio de Michoacán.

Real Academia Española (1984). Diccionario de Autoridades. Madrid: Gredos.

Ricoeur, P. (2001). La metáfora viva. España: Ediciones cristiandad.

Universidad Nacional Autonoma de Mexico. (1991). Legislacion Universitaria. Mexico: UNAM.

Uribe, O. (1984). Por una auténtica renovación universitaria. México: IIS/UNAM. 